\title{
Oringual syes \\ Gastrointestinal bleeding in acute pancreatitis: etiology, clinical features, risk factors and outcome
}

\author{
Surinder S Rana ${ }^{1}$, Vishal Sharma ${ }^{1}$, Deepak K Bhasin ${ }^{1}$, Ravi Sharma ${ }^{1}$, \\ Rajesh Gupta ${ }^{2}$, Puneet Chhabra', Mandeep Kang ${ }^{3}$
}

\begin{abstract}
Department of Gastroenterology ${ }^{1}$, Surgery $^{2}$ and Radiodiagnosis ${ }^{3}$, Post Graduate Institute of Medical Education and Research (PGIMER), Sector 12, Chandigarh - 160012, India

\section{Correspondence:}

Dr. Surinder Singh Rana

Email:drsurinderrana@yahoo.co.in

Background: There is paucity of data on the effect of interventions on risk of gastrointestinal bleeding (GIB) in acute pancreatitis (AP). Methods: Retrospective study of records of patients with AP and GIB. Results: 16 (3.7\%) patients (14 males; mean age 39.3 \pm 12.8 years) had gastrointestinal bleeding. Two patients had peptic ulcer disease related GIB. The cause of GIB in remaining patients was: pseudoaneurysms in 5, gastrointestinal fistulization in 4 , and no identifiable lesion in 5. Two patients with pseudoaneurysms were treated with angioembolisation whereas 3 needed surgery. The patients with gastrointestinal fistula had complicated course and 3 of these 4 patients died due to sepsis and multi organ failure. Rest 5 patients with no identifiable lesion were managed conservatively and there was no recurrence of GIB. Of the 14 patients with AP related GIB, a previous intervention had been done in $11(79 \%)$ patients. Fifty-three patients $(12.7 \%)$ without GIB died whereas $5(31.2 \%)$ patients with GIB succumbed to the illness $(\mathrm{p}=0.04)$. Conclusions: The majority of our patients of AP with GIB had antecedent history of interventions. The mortality was higher in patients with GIB, which was not due to hemorrhage but to sepsis and related complications.
\end{abstract}

KEYWORDS: acute pancreatitis; bleeding; pancreatic necrosis

\section{Introduction}

Acute pancreatitis (AP) is a perplexing gastrointestinal disease with variable degree of severity, course and outcome. Necrotizing pancreatitis is a more severe form of AP associated with pancreatic parenchymal necrosis and/or peri-pancreatic necrosis. ${ }^{1}$ Gastrointestinal bleeding (GIB) is an infrequent but potentially fatal complication of AP. It was considered a marker of severity in the original Atlanta classification but was removed in the revised version as it is a late manifestation and does not represent organ failure. ${ }^{1,2}$ A recent large population-based study demonstrated that although organ failure had more adverse effects on the outcome of AP than did GIB, the bleed still modestly increased the risk of mortality as well as the length of the hospital stay in patients with AP. ${ }^{3}$

The cause of GIB in acute pancreatitis can be varied and includes non-pancreatic causes like peptic ulcer disease, aggravated or induced by stress and nonsteroidal antiinflammatory drug use for pain relief or due to local vascular complications of acute pancreatitis like thrombosis of the portal 
venous system leading to formation of varices, erosions of the upper abdominal arteries by the inflammatory process or by the collections, formation of pseudoaneurysms or fistulization into the gastrointestinal tract. ${ }^{3-5}$ The combination of severe necrotizing pancreatitis and massive gastrointestinal bleed can be a therapeutic challenge and is associated with high mortality. ${ }^{6,7}$

Because of the rarity of GIB in AP, its risk factors have been infrequently studied. The presence of pancreatic necrosis, sepsis, collection and organ failure might be more common in bleeders compared with non-bleeders. ${ }^{5}$ The impact of surgical and radiological interventions of local complications of AP on the risk of GIB has also rarely been studied. One study reported increased frequency of bleeding in patients who underwent early surgical intervention as compared to patients with delayed intervention. ${ }^{8}$ However, the impact of percutaneous interventions in increasing the risk of GIB in AP is not clear. We retrospectively studied the etiology, clinical and endoscopic features, impact of prior interventions and outcome of our patients with AP having GIB.

\section{Patients and Methods}

The present study was a retrospective analysis of AP patients with GIB seen at our unit at a large tertiary care referral teaching hospital in North India over the last four years. The diagnosis of acute pancreatitis was based on the presence of two of the three features including presence of typical abdominal pain, elevation of serum amylase or lipase to more than thrice the upper limit of normal or presence of radiological evidence of acute pancreatitis. ${ }^{1}$ We noted the clinical details including duration of pain, onset to bleeding, age, gender, and etiology of acute pancreatitis as well the hematological and biochemical investigations and endoscopic findings. Details of the invasive procedure prior to bleed including insertion of percutaneous drains (PCD) and surgery were recorded. The outcome i.e. sepsis, organ failure, recurrence of bleeding and mortality was noted. The details of the therapeutic interventions done for hemostasis were also retrieved and noted. The various definitions used in the study include:

Pancreatic necrosis: The presence of non-enhancing areas in the pancreas as noted on contrast enhanced computed tomography scan.

Severity of acute pancreatitis: Severity of AP was defined using revised Atlanta definitions where the presence of persistent organ failure ( $>48$ hours) was the central feature of severe acute pancreatitis. ${ }^{1}$ Presence of local complications and/ or transient organ failure ( $<48$ hours) defined moderately severe AP and absence of local/systemic complications and organ failure signified mild AP.

\section{Assessment of gastrointestinal bleeding}

We recorded the presentation of bleed (hematemesis, melena), presence or absence of hemodynamic compromise, need for transfusion, and results of evaluation for the cause of bleeding including endoscopy and computed tomography guided angiography. All patients received standard therapy including intravenous proton pump inhibitors, intravenous fluids and correction of coagulopathy and thrombocytopenia, if present, by transfusion of appropriate blood component. Death, discharge and readmission were recorded. The management of pancreatitis was as per standard guidelines including analgesia, management of organ dysfunction, antibiotics on suspicion of infection and drainage of infected collections using radiology guided pigtails.

\section{Statistical analysis}

The descriptive data was presented as percentages for categorical variables and mean $\pm \mathrm{SD}$ for quantitative variables. The continuous variables were compared using student t-test whereas the categorical variables were compared using the Chi-square test. A p value of $<0.05$ was considered significant.

\section{Results}

Of 432 patients with AP seen at our unit over the last four years, $16(3.7 \%$ ) patients (14 males; mean age $39.3 \pm 12.8$ years) had gastrointestinal bleeding. All our patients presented with overt gastrointestinal bleeding and hemodynamic instability was seen in $13(81 \%)$ patients. Blood transfusion was required in all patients. The etiology of acute pancreatitis was alcohol in $11(68.75 \%)$, gall-stones in $1(6.25 \%)$, blunt abdominal trauma in $1(6.25 \%)$, and idiopathic in $3(18.75 \%)$ patients. These patients had GIB 3-12 weeks after the onset of AP. Twelve (75\%) patients had severe AP whereas 4 patients had moderately severe AP. None of the patients had mild AP. All patients had pancreatic necrosis on computed tomography (Table $\mathbf{1}$ ).

Upper gastrointestinal endoscopy revealed duodenal ulcer in 1 , and severe esophagitis in 1 patient and these two patients were successfully treated with proton pump inhibitors and 
Table 1:Details of 16 patients with acute necrotizing pancreatitis and gastrointestinal bleed

\begin{tabular}{|c|c|c|c|c|c|c|}
\hline$\overline{\text { Age }}$ & Sex & Etiology & Preceding Intervention & Cause & Pseudoaneurysm Location & Death \\
\hline$\overline{18}$ & $\mathrm{M}$ & Idiopathic & $\mathrm{N}$ & Pseudoaneurysm & Splenic & No \\
\hline 60 & $\mathrm{~F}$ & GSD & $\mathrm{P}$ & Pseudoaneurysm & Left hepatic & No \\
\hline 50 & M & Alcohol & $\mathrm{N}$ & Pseudoaneurysm & Splenic & No \\
\hline 38 & M & Alcohol & $\mathrm{P}, \mathrm{S}$ & Fistula & Duodenum & No \\
\hline 52 & M & Alcohol & $\mathrm{P}, \mathrm{S}$, & Fistula & Duodenum & Yes \\
\hline 32 & $\mathrm{~F}$ & Idiopathic & $\mathrm{N}$ & Pseudoaneurysm & Splenic & No \\
\hline 46 & M & Alcohol & $\mathrm{P}$ & Fistula & Stomach & Yes \\
\hline 34 & M & Trauma & $\mathrm{P}$ & Not Identified & No & Yes \\
\hline 42 & M & Alcohol & $\mathrm{P}, \mathrm{S}$ & Not Identified & No & No \\
\hline 21 & M & Alcohol & $\mathrm{P}$ & Pseudoaneurysm & Transverse mesocolic & Yes \\
\hline 61 & M & Alcohol & $\mathrm{P}$ & Fistula & Duodenum & Yes \\
\hline 28 & M & Alcohol & $\mathrm{T}$ & Not Identified & No & No \\
\hline 28 & M & Idiopathic & $\mathrm{T}$ & Not Identified & No & No \\
\hline 46 & M & Alcohol & $\mathrm{N}$ & Duodenal Ulcer & No & No \\
\hline 32 & M & Alcohol & $\mathrm{N}$ & Esophagitis & No & No \\
\hline 42 & M & Alcohol & $\mathrm{P}, \mathrm{S}$ & Not Identified & No & No \\
\hline
\end{tabular}

M: Male, F: Female; P: Percutaneous drainage, S: Surgery, T: Transmural drainage; D: Digital Subtraction angiography; N: None; GSD:

Gall stone disease

endoscopic intervention (Figure 1). Although 4 (25\%) patients had splenic vein thrombosis, none of them had esophageal or gastric varices. The remaining 14 patients had AP related GIB and endoscopy revealed fistulous opening in the duodenum and stomach in 3 and 1 patient, respectively (Figure 2). Necrotic material with granulation tissue was seen from the fistulous opening in the gastrointestinal lumen. Although the bleed was self-limiting in all 4 patients but these patients had a complicated course, and all required surgery for infected necrosis and 3 of these 4 patients died due to sepsis and multiorgan failure.

Upper gastrointestinal endoscopy did not identify any bleeding lesion in the remaining 10 patients. All these 14 patients also underwent computed tomographic (CT)

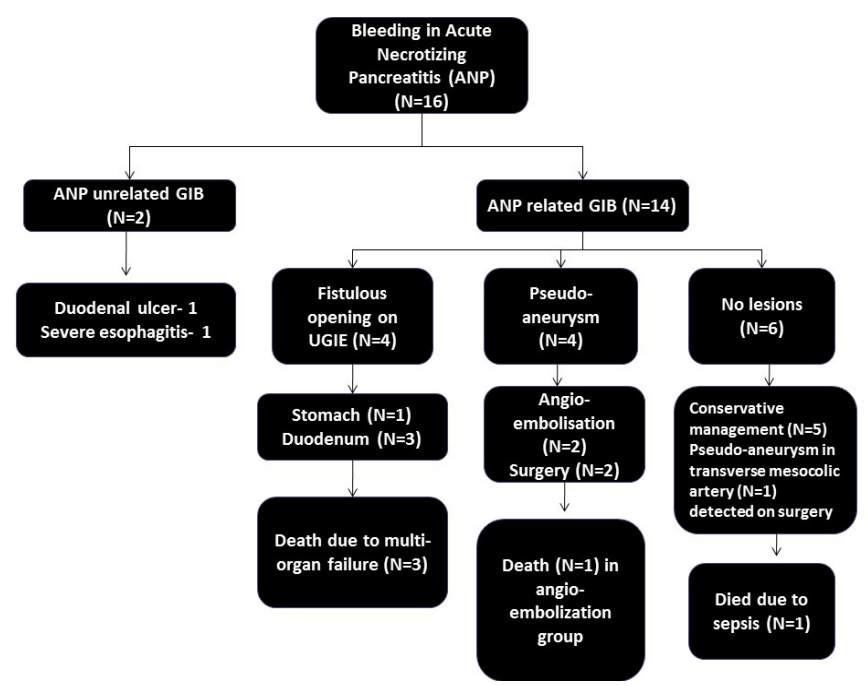

Figure 1: The course of sixteen patients with gastrointestinal bleed angiography and pseudoaneurysms were identified in 4 patients (splenic artery in 3 and left hepatic artery in 1). Two of the 4 patients with pseudoaneurysms were successfully treated with angioembolisation whereas 2 needed immediate surgery because of hemodynamic compromise. One patient initially treated by angioembolisation needed surgery for worsening sepsis and succumbed to the illness.

Of the remaining 6 patients, 1 patient was operated on because of hemodynamic compromise and intra operatively pseudoaneurysm of transverse mesocolic artery could be identified and successfully ligated. However, this patient died due to sepsis and organ failure. The remaining 5 patients with no identifiable lesions were managed conservatively and there was no recurrence of GIB in these patients.

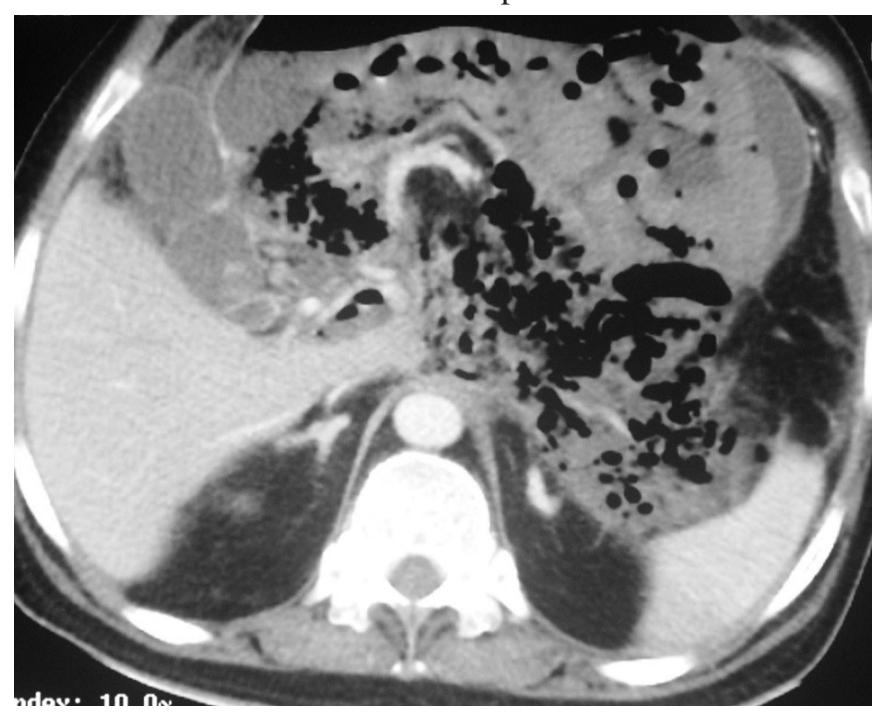

Figure 2: CT showing extensive emphysematous changes in patient with fistulization of the collection in the stomach. 


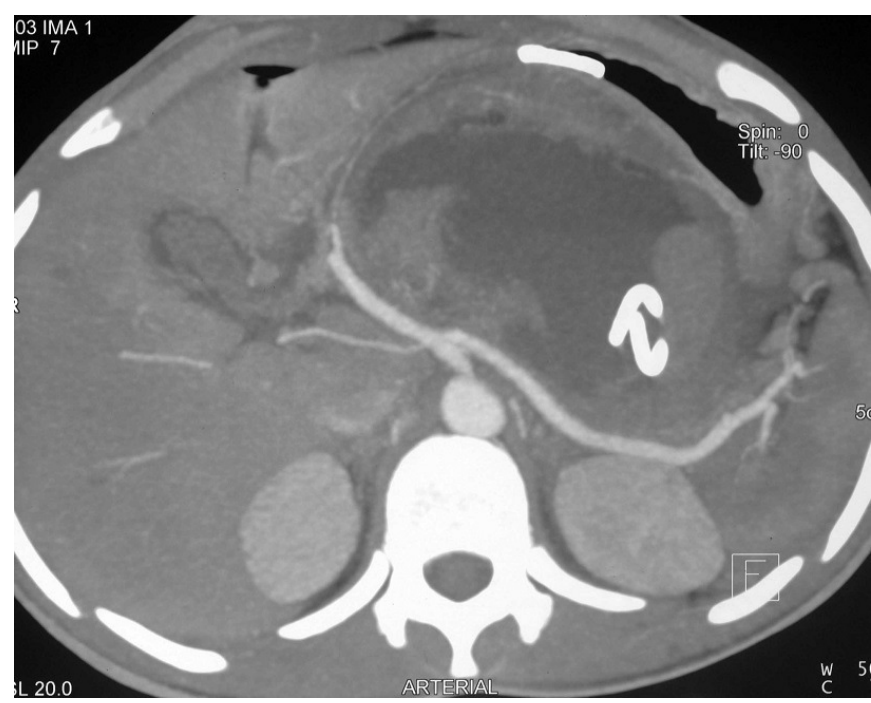

Figure 3: CT in patient with self limiting bleed after endoscopic transmural drainage of walled off necrosis showing normal major arteries. Hyperdense contents in the collection suggestive of blood. Also seen are transmural stents.

\section{Impact of previous intervention on GIB}

One hundred and twenty two (28.2\%) patients with AP during the study period needed endoscopic/radiological or surgical intervention and 11 (9\%) of these had GIB. On the other hand, $3(0.9 \%)$ of patients without any intervention had GIB due to pancreatitis related causes $(\mathrm{p}=0.0001)$. Of these 11 patients, the type of intervention was percutaneous drainage in 9 , endoscopic drainage in 2 and surgery in 4 patients and the bleed occurred 2-16 days after the intervention (Figure 3). The Odd's ratio for intervention as a factor for GIB was 6.04 (95\% CI: 2.05 to $17.78, \mathrm{P}=0.0011$ )

\section{Outcome}

Fifty-three of 416 patients (12.7\%) without GIB died whereas 5 of $16(31.2 \%)$ patients with GIB succumbed to the illness $(\mathrm{p}=0.04)$. The mortality in patients with acute necrotizing pancreatitis but without GIB was $16.2 \%$ and although this was lower than the patients with ANP and GIB the difference was not statistically significant. Also, the cause of mortality was not related directly to gastrointestinal bleeding but to the occurrence of sepsis and organ failure as hemostasis was achieved in all patients. The patients with gastrointestinal fistulae and pseudoaneurysms had an adverse outcome with $75 \%$ and $40 \%$ patients succumbing to the illness.

\section{Discussion}

Acute pancreatitis is a disease of varying severity with local and systemic complications accompanying severe pancreatitis. ${ }^{1}$ GIB is one of the potentially fatal but rare complications of AP. Also, management of infected or symptomatic collections needs interventions in the form of percutaneous drains or endoscopic/surgical necrosectomy/ drainage. ${ }^{9,10}$ It is not clear if these interventions predispose to hemorrhagic complications in such patients. Systematic studies on gastrointestinal bleeding in acute pancreatitis are few and have not addressed the issue of intervention predisposing to occurrence of bleeding. In this single center study, we assessed the frequency, etiology, risk factors and outcome of GIB in AP.

The exact frequency of GIB in AP is not clear but various studies have reported the frequency of fatal hemorrhagic complications varying from $1.2 \%$ to $14.5 \% .^{3,6,11}$ One study from another tertiary care center in north India reported the frequency of GIB to be $3.6 \%$ and this is similar to the frequency reported by us. The GIB in AP could be due to non-pancreatic causes like peptic ulcer disease or local vascular complications of acute pancreatitis like venous thrombosis leading to the formation of varices, erosions of the upper abdominal arteries by the inflammatory process or by the collections, and formation of pseudoaneurysms or fistulas into the gastrointestinal tract. ${ }^{3-}$ ${ }^{5}$ In our study, $13 \%$ patients had GIB due to peptic ulcer disease whereas $31 \%$ had arterial bleed due to pseudo aneurysm formation and $25 \%$ through the formation of fistulas into the gastrointestinal tract. In $31 \%$ patients no definite cause for GIB could be identified. A literature review reported arterial bleeding in $30 \%$ and venous bleeding in $34 \%$ patients. ${ }^{6}$ Sharma et al reported the coexistent esophageal and/or gastroduodenal ulcers to be the major cause of GIB in patients with AP $(81.2 \% \text { patients })^{5}$. Other causes of GIB were spontaneous rupture of pseudocyst into the duodenum in $12.5 \%$ patients, and gastric variceal bleeding secondary to splenic vein thrombosis in one patient. ${ }^{5}$ These collections occurring in the course of ANP possibly represented walled off necrosis and not pseudocyst per se. In contrast to this report, none of our patients had variceal bleed.

The risk factors for GIB in AP have also been infrequently studied. Presence of pancreatic necrosis especially infected necrosis, sepsis, collections, prior surgical intervention and organ failure are important risk factors for GIB in AP. ${ }^{5,6}$ One study reported bleeding to be more common in infected 
necrosis as compared to sterile necrosis. ${ }^{12}$ In our study, GIB was more common in patients with prior intervention as compared to patients with no history of intervention and all the patients with GIB had necrotizing pancreatitis. It is not clear if the intervention itself increased the risk of bleeding or the intervention represented more severe disease with a higher risk of attendant local complications and erosion of surrounding vessels. One retrospective report suggested that the presence of severe ischemic changes correlated with a higher Ranson's score. ${ }^{13}$ It has also been suggested that drainage catheters should be soft and placed away from major vessels to avoid bleeding. ${ }^{6}$

The impact of GIB on the course of AP is still not clear. GIB was initially considered a marker of severity in the original Atlanta classification but was removed in the revised version as it was found to be a late manifestation and did not represent any organ failure of. ${ }^{1,2}$ Some studies have reported mortality rate as high as $50 \%$ in patients with GIB whereas others have reported that hemorrhagic complications have little bearing on mortality. ${ }^{5,6}$ A recent large population-based study demonstrated that GIB modestly increased the risk of mortality as well as the length of the hospital stay in patients with AP. ${ }^{3}$ In our study, the mortality in patients with GIB was significantly higher than the mortality in patients without GIB. When all patients of acute necrotizing pancreatitis were compared, the mortality was still higher in patients with GIB but the difference was not statistically significant. Also, the mortality was not related to hemorrhage per se but resulted from occurrence of sepsis and related complications and this observation is similar to previous studies. ${ }^{1,5,6}$ Gastrointestinal fistula related bleeding had the highest mortality followed by bleeding due to pseudoaneurysms.

To conclude, a majority of our patients of acute pancreatitis with gastrointestinal bleeding had antecedent history of interventions. It is not clear if the occurrence of bleeding represents a complication of the interventions or is merely a marker of severe disease. The mortality is higher in patients with GIB; the mortality however was not due to pseudoaneurysmal hemorrhage but resulted from occurrence of sepsis and related complications.

\section{References}

1. Banks PA, Bollen TL, Dervenis C, Gooszen HG, Johnson CD, Sarr MG, et al. Classification of acute pancreatitis-2012: revision of the Atlanta classification and definitions by international consensus. Gut 2013;62:102-11.

2. Bradley EL, 3rd. A clinically based classification system for acute pancreatitis. Summary of the International Symposium on Acute Pancreatitis, Atlanta, Ga, September 11 through 13, 1992. Arch Surg 1993;128:586-90.

3. Shen HN, Lu CL, Li CY. The effect of gastrointestinal bleeding on outcomes of patients with acute pancreatitis: a national population-based study. Pancreatology. 2012;12:331-6.

4. Bhasin DK, Rana SS, Sharma V, Rao C, Gupta V, Gupta R, et al. Non-surgical management of pancreatic pseudocysts associated with arterial pseudoaneurysm. Pancreatology. 2013;13:250-3.

5. Sharma PK, Madan K, Garg PK. Hemorrhage in acute pancreatitis: should gastrointestinal bleeding be considered an organ failure? Pancreas. 2008;36:141-5.

6. Flati G, Andrén-Sandberg A, La Pinta M, Porowska B, Carboni M. Potentially fatal bleeding in acute pancreatitis: pathophysiology, prevention, and treatment. Pancreas. 2003;26:8-14.

7. Dörffel T, Wruck T, Rückert RI, Romaniuk P, Dörffel Q, Wermke W. Vascular complications in acute pancreatitis assessed by color duplex ultrasonography. Pancreas. 2000;21:126-33.

8. Kriwanek S, Gschwantler M, Beckerhim P, Armbruster C, Roka R. Complications after surgery for necrotizing pancreatitis: risk factors and prognosis. Eur J Surg. 1999;165:952-7.

9. Rana SS, Bhasin DK, Sharma RK, Kathiresan J, Gupta R. Do the morphological features of walled off pancreatic necrosis on endoscopic ultrasound determine the outcome of endoscopic transmural drainage? Endosc Ultrasound. 2014;3:118-22.

10. Babu RY, Gupta R, Kang M, Bhasin DK, Rana SS, Singh R. Predictors of surgery in patients with severe acute pancreatitis managed by the step-up approach. Ann Surg. 2013;257:737-50.

11. Flati G, Andren-Sandberg A, La Pinta M, Porowska B, Carboni M. Potentially fatal hemorrhage in acute pancreatitis: pathophysiology, prevention andtreatment. Pancreas. 2003;26:8-14.

12. Tsiotos GC, Juarez Munoz M, Sarr MG. Intraabdominal haemorrhage complicating surgical management of necrotizing pancreatitis. Pancreas. 1996;12:126-30.

13. Inoue K, Hirota M, Beppu T, Ishiko T, Kimura Y, Maeda K, et al. Angiographic features in acute pancreatitis: the severity of abdominal vessel ischemic change reflects the severity of acute pancreatitis. JOP. 2003;4:207-13. 